\title{
Tachistoscopic applications of Micro Experimental Laboratory (MEL) used with IBM PC compatibles: Stimulus and response timing issues
}

\author{
KARIN MOGG and BRENDAN P. BRADLEY \\ University of Cambridge, Cambridge, England
}

\begin{abstract}
IBM PCs and compatibles are increasingly used in psychological research for tachistoscopic tasks, which require precise control of timing of stimulus durations and accurate measurement of response latencies. The application of MEL in such research is discussed; several precautions which influence stimulus timing are noted, and some data on measurement error with keypress responses are presented.
\end{abstract}

We have used Micro Experimental Laboratory (MEL; Schneider, 1988, 1990) Version 1.0 in several experiments to present brief masked visual stimuli in our research into emotion and cognition (e.g., Bradley, Mogg, Millar, \& White, 1995; Bradley, Mogg, \& Williams, 1994; Mogg, Bradley, \& Williams, 1995; Mogg, Bradley, Williams, \& Mathews, 1993). In parallel with this work, we have also conducted a series of studies using more traditional methodology, such as a three-field projection tachistoscope, to present short-duration visual stimuli (e.g., Bradley, Mogg, Millar, Bonham-Carter, et al., 1995). Consequently, we have been able to compare directly the relative merits of mechanical versus computerized tachistoscopic methods. The aim of this paper is to describe some issues which we have encountered while using MEL for brief visual displays, as we hope this will assist others in similar lines of research.

First, it is necessary to briefly describe the equipment, as this partly determines the nature of the screen display. We have largely used the IBM PS/2 Model 30286 or a Toshiba T3100SX 386 , each with IBM 8513 or 8512 color monitors. The latter have a refresh rate of $70 \mathrm{~Hz}$, which indicates that the screen display is being refreshed 70 times per second (i.e., once every $14.3 \mathrm{msec}$ ). Thus, the minimum stimulus onset asynchrony (SOA; i.e., interval between the onset of one stimulus and the onset of the next) is $14.3 \mathrm{msec}$. The phosphor decay rates for modern computer screens are fast in order to minimize image smear, and the decay times (to $10 \%$ point) for many common phosphors are less than $1 \mathrm{msec}$ (Travis, 1991). We checked the characteristics of our IBM screen displays using an oscilloscope (Gould

Our research work is supported by the Wellcome Trust and Medical Research Council. Our grateful thanks are extended to Brian Grainger, University of Cambridge Computer Laboratory, for his advice and for designing and building the keypress simulator, and also to John Baldwin and Hubert Jackson for technical support. Correspondence should be addressed to K. Mogg, Department of Experimental Psychology, University of Cambridge, Downing Street, Cambridge CB2 3EB, England (fax: 44-223-333564).
Storage $054100 ; y$-axis dc coupled) and a light-sensitive diode with a preamplifier and ultraviolet and infrared filters (used to restrict the spectrum to the visible wavelengths), and found that the duration of the display of a stimulus (such as a word or row of crosses in the center of the screen) appeared to be approximately $1 \mathrm{msec}$, which was repeated (i.e., refreshed) every $14.3 \mathrm{msec}$ (an example of output from one of our display tests is given later). Thus, the normal "continuous" display of a stimulus comprises repeated displays, which seem to decay largely within $1 \mathrm{msec}$, with a $14.3-\mathrm{msec}$ SOA (this finding does not seem to be specific to these IBM monitors, as we obtained similar results from our Viglen IV/25 486 with a Viglen CA1428-LE monitor).

In running tachistoscopic experiments, MEL may be used to display a stimulus (such as a word) followed by a mask (such as a random letter string) with a word-mask SOA of $14.3 \mathrm{msec}$ (e.g., Mogg et al., 1993). In the absence of a mask, the apparent duration of the stimulus will vary considerably, due to variations in visual persistence (Felsten $\&$ Wasserman, 1980). The use of a mask allows more precise control over the effective duration of the preceding stimulus word by terminating effects of visual persistence on the stimulus. Consequently, in tachistoscopic experiments such as ours, the word-mask SOA is of greater importance than the objective duration of the word stimulus.

However, to achieve such brief stimulus-mask SOAs with MEL, several precautions should be noted. First, in accordance with the MEL manual instructions (Schneider, 1990), the WAITTOP instruction should be applied to the FRAME presenting the stimulus word and the subsequent FRAME (the mask). This is important in order to coordinate the timing of the stimulus and mask displays with each other and also with the start of response timing (if WAITTOP is not used, there may be variation in timing of up to one screen refresh interval). Second, the ERASE instruction should not be used on the FRAME presenting the stimulus or the subsequent FRAME, because this can interfere with the timing of very brief displays and may result in the stimulus's not 
being shown at all, or being shown intermittently over a number of trials. Third, to obtain the minimum stimulusmask SOA, the DURATION instruction for the stimulus word should be less than the refresh interval of the monitor. Thus, to achieve a $14.3-\mathrm{msec}$ SOA, we typically set the duration of the stimulus FRAME to 1 or $10 \mathrm{msec}$ (we have found that either duration instruction gives similar results provided that ERASE YES is not used on the frame subsequent to the brief stimulus display). If the DURATION instruction is set to $14 \mathrm{msec}$, the presentation of the stimulus word (assessed with an oscilloscope and diode) tends to vary across trials. That is, sometimes the word is shown once with a word-mask SOA of $14.3 \mathrm{msec}$ and sometimes the word is shown twice before the mask so that the wordmask SOA is effectively $28.6 \mathrm{msec}$.

The latter problem similarly occurs for DURATION instructions that are multiples of the refresh interval. Thus, to achieve a consistent word-mask SOA of $42.9 \mathrm{msec}$, the DURATION instruction for the word should be less than $42 \mathrm{msec}$ and greater than $29 \mathrm{msec}$. This range of values would result in the word's being shown three times, once every $14.3 \mathrm{msec}$, and the mask on the subsequent FRAME would then be presented approximately $43 \mathrm{msec}$ after the initial onset of the word display. (By contrast, a duration instruction of $43 \mathrm{msec}$ results in the word's being presented sometimes three times and sometimes four times before the mask, so that the word-mask SOA is variable across a series of trials-a mixture of 42.9- and 57.2-msec SOAs). It should also be noted that to use such short durations it is necessary to set the instruction WARN ON DURATION NOT MULTIPLE OF REFRESH TIME on the DEFAULT form to NO.

A problem with research using subthreshold stimuli is ensuring that the stimuli (which cannot be "seen" by the experimenter) are being reliably presented. Thus, it seems advisable that researchers who wish to produce tachistoscopic displays with MEL (or any other software package) should check that the monitor is presenting the stimuli for the desired SOAs and that the SOAs are consistent across a number of trials. The output of such a check on a single trial, using an oscilloscope and light-sensitive diode, is illusirated in Figure 1, which shows the display of a word (the first spike on the oscilloscope trace), followed by a letter mask (the second spike), with a word-mask SOA of $14.3 \mathrm{msec}$. When conducting such checks for a series of trials, we have also included, for purposes of comparison, trials on which the FRAME instructions were modified such that a space (i.e.,' ') was displayed instead of a word; the resultant display from such a trial would be a single spike (i.e., the mask alone). (The use of this stimulus arrangement, where half the trials consist of a word and mask and half consist of a mask alone, is similar to that used in an awareness-check task that assesses whether or not subjects can discriminate the presence of the masked word stimuli; see, e.g., Mogg et al., 1995.)

Another simple preexperimental check, which does not require additional equipment, can be carried out to establish that the subthreshold stimuli are being reliably presented. On each trial, the word can be displaced a line or two above the mask so that it now becomes clearly visible
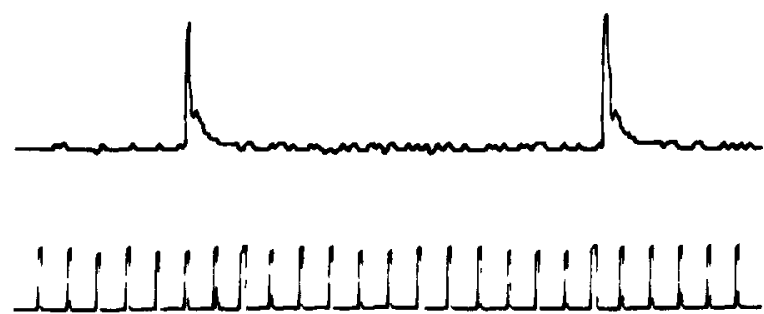

Figure 1. Oscilloscope trace of the screen display of a word (first spike) followed by a letter mask (second spike) presented with a 14.3-msec SOA. Lower trace is calibration scale in $1-\mathrm{msec}$ intervals.

(the word display will persist with ERASE set to NO). Thus, it is possible to check easily and rapidly, for a large number of trials, whether or not the word stimuli are being consistently presented. (For further details of precautions for using brief visual displays, see Schneider, 1990.)

Another of our concerns regarding the use of MEL has been the accuracy of measurement of response times (RTs) when using the keyboard. (We have had considerable difficulty with the supply of MEL response boxes in recent years and have managed to obtain only one, Version 5.0, which we have used in several tasks, such as a modified Stroop color-naming task, following a minor modification to increase the sensitivity of the voice key; see, e.g., Bradley, Mogg, Millar, \& White, 1995; Mogg et al., 1993.) To assess error variance of RTs obtained with MEL using an IBM-compatible keyboard, we employed a purpose-built electronic keypress simulator (which was designed and built by Brian Grainger, University of Cambridge Computer Laboratory). The keypress simulator was connected at one end to a key (u) on a Viglen keyboard (Model DFK2020UK) and at the other end to a light-sensitive diode that was attached to the screen (IBM 8513 monitor controlled by an IBM PS/2 Model 30286 computer). The keypress simulator detected the onset of a visual stimulus on the screen, via the light-sensitive diode, and simulated a keypress through an electronic switch that was connected across the conventional key switch at the back of the keyboard. The delay, within the keypress simulator, between the detection of the stimulus and the onset of the simulated keypress was less than $100 \mu \mathrm{sec}$. The duration of the simulated keypress was 100 msec.

A simple MEL program was written to present, on each trial, the following sequence: (1) a blank screen (DURATION instruction $=500 \mathrm{msec} ;$ ERASE $=$ YES; TERMINATE $=$ TIMEOUT; DISPLAY TYPE = WAITTOP), followed by (2) the target stimulus- - a row of 10 uppercase crosses, i.e., XXXXXXXXXXwhich was displayed in the middle of the screen until a keypress response was made or, in the absence of a response, for $1,000 \mathrm{msec}$ (START LINE = 13; DURATION = 1,000 msec; ERASE $=$ NO; TERMINATE $=$ RESPONSE; DISPLAY TYPE $=$ WAIT TOP), followed by (3) an intertrial interval (DURATION = $100 \mathrm{msec} ;$ ERASE $=$ NO; TERMINATE $=$ TIMEOUT; DISPLAY TYPE = WAITTOP). We placed the stimulus in the middle of the screen (START LINE 13), as this position is indicated for reaction-time tasks in the MEL manual. There were 1,000 
trials in each test session; the program was run five times to provide RT data from 5,000 trials.

The results showed that the mean RT was $14.1 \mathrm{msec}$, the standard deviation was $0.6 \mathrm{msec}$, and the range was $12-15 \mathrm{msec}$ (all 5,000 trials were coded as correct responses; that is, RESPAC $=1$ ). The distribution of these data is shown in Figure 2. The bulk of the RT data (i.e., 99.92\%) had a random error of $\pm 1 \mathrm{msec}$ around the median of 14 msec.

Thus, there was an average delay of approximately $14 \mathrm{msec}$ between the start of the stimulus FRAME and the detection of a keyboard response. This delay depends not only on relatively constant factors associated with the hardware and software, such as the scan rate of the particular keyboard used, but also on experimental variables such as the position of the stimulus on the screen. For example, we checked the delay for 5,000 trials with the stimulus appearing at the top rather than the middle of the screen (i.e., START LINE was 1, instead of 13). The mean delay between the start of the stimulus FRAME and detection of a keyboard response was $7.6 \mathrm{msec}(S D=1.0$, range $5-10 \mathrm{msec}$; for $99.52 \%$ of trials, the range was $6-9 \mathrm{msec}$, i.e., $\pm 1.5 \mathrm{msec}$ ). The difference in mean delay of $6.5 \mathrm{msec}$ between the two conditions (i.e., $7.6 \mathrm{msec}$ vs. $14.1 \mathrm{msec}$ ) represents the time taken for the raster scan to move from Line 1 to Line 13 of the display.

We found no evidence that the choice of response key influenced the performance of this particular hardware combination (i.e., Viglen DFK2020UK keyboard with IBM PS/ 2 Model 30286 computer). For example, with the stimulus on the top line (i.e., START LINE $=1$ ), the mean delay between the start of the stimulus FRAME and the keyboard response for keys u, q, and z, respectively, was 7.6, 7.7 , and $7.6 \mathrm{msec}(S D=1.0 \mathrm{msec}$ for each of these tests; $N=5,000$ trials).

We also found that the choice of computer did not appear to significantly affect performance. For example, we also evaluated the Viglen DFK2020UK keyboard with a Viglen IV/25 486 computer. With the stimulus in the middle of the screen (START LINE $=13$ ) and the response measured from key $u$, the mean delay between the start of the stimulus FRAME and the detection of a keyboard response was $14.1 \mathrm{msec}(S D=1.0$, range $=12-16 \mathrm{msec} ; N=5,000$ trials) for the Viglen IV/25 computer, which is similar to the results reported above for the same keyboard used with the IBM PS $/ 2(M=14.1 \mathrm{msec}$, range $=12-15 \mathrm{msec})$.

These mean delays, which result from factors such as the transit time of the raster scan, are not necessarily a major problem for research into individual differences, as they are typically a constant factor across subjects. However, it is important to ensure that any sources of random error variance be kept to a minimum.

We have used our electronic keypress simulator to compare the mean delays and random errors of different response devices, which are summarized in Table 1. The top half of the table illustrates the variation in performance found between three different models of the Viglen keyboard. Each was tested in combination with a Viglen IV/ 25 computer (an IBM PS/ 2 was not used in this series

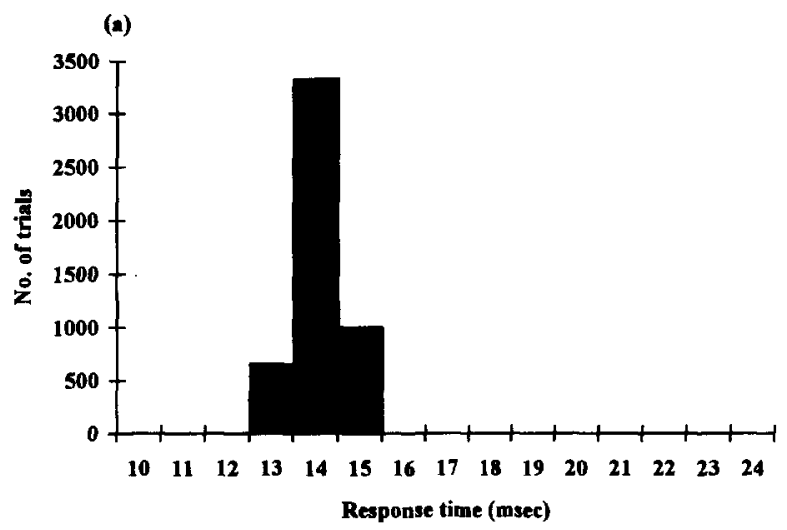

(b)

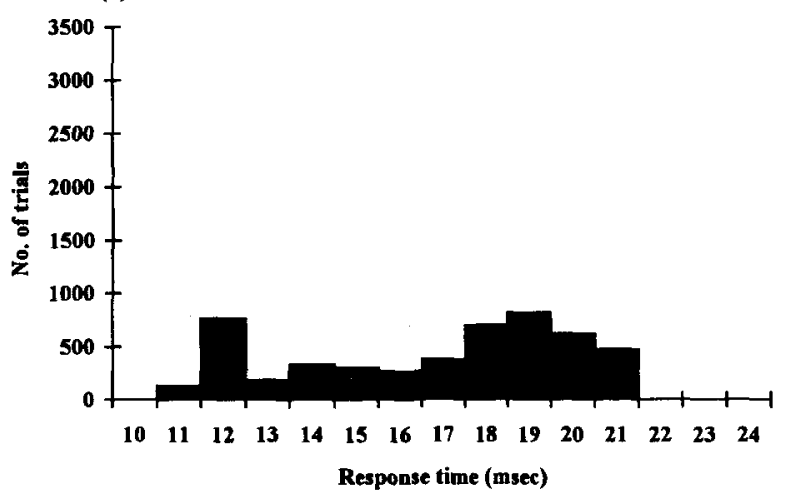

(c)

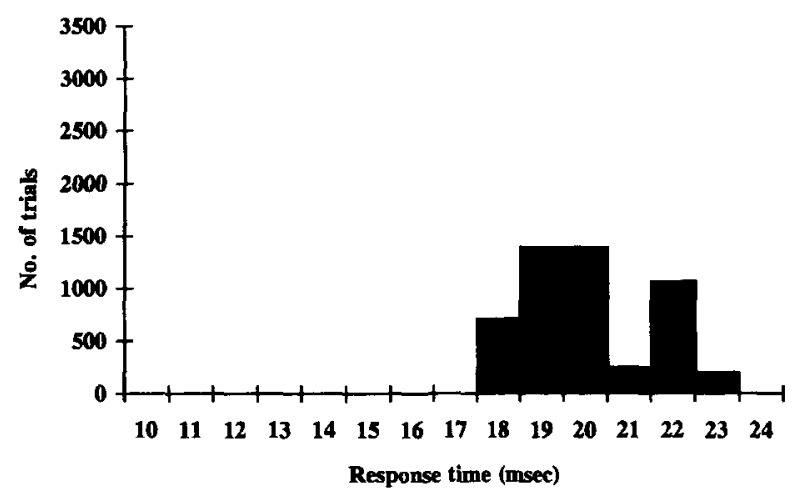

Figure 2. Frequency distribution of response time (interval between start of the stimulus FRAME and detection of keyboard response) for (a) Viglen DFK2020UK keyboard, (b) IBM PS/2 1391406 keyboard, and (c) MEL response box Version 5.0; each used with an IBM PS/ 2 Model 30286 computer $(N=5,000$ trials; stimulus displayed in middle of screen, i.e., START LINE 13 in MEL).

of tests, owing to compatibility problems with the Viglen DFK2020SUK104 keyboard). There were notable differences between the three keyboards, with the Viglen DFK2020UK showing the smallest degree of random error (all data were within $\pm 2 \mathrm{msec}$ of the median $14 \mathrm{msec}$ ). Thus, this keyboard appears to be particularly suitable for use in RT experiments. Unfortunately, this model is no longer in production and the more recent Viglen keyboard Model 6312 performed rather less well (99.2\% data within $\pm 6 \mathrm{msec}$ of the median $17 \mathrm{msec}$ ). 
Table 1

Mean Interval, Standard Deviation, and Range (in Milliseconds) Between Start of Stimulus FRAME and Detection of a Keyboard Response, Recorded by MEL for Various Computers and Response Devices

\begin{tabular}{|c|c|c|c|c|c|c|c|}
\hline Computer & Response Device & Key & $\begin{array}{c}\text { No. } \\
\text { Trials }\end{array}$ & $\begin{array}{c}\text { No. } \\
\text { Errors/ } \\
\text { Outliers }\end{array}$ & $\begin{array}{c}\text { Mean } \\
\mathrm{RT}\end{array}$ & $S D$ & Range \\
\hline Viglen IV/25 486 & $\begin{array}{l}\text { Viglen keyboard DFK2020UK } \\
\text { Viglen keyboard DFK2020SUKF } 104 \\
\text { Viglen keyboard } 63: 2\end{array}$ & $\begin{array}{l}u \\
u \\
u\end{array}$ & $\begin{array}{l}5,000 \\
5,000 \\
5,000\end{array}$ & $\begin{array}{l}0 \\
3 * \\
1 \dagger\end{array}$ & $\begin{array}{l}14.1 \\
14.8 \\
16.2\end{array}$ & $\begin{array}{l}1.0 \\
2.8 \\
2.8\end{array}$ & $\begin{array}{r}12-16( \pm 2) \\
6-19( \pm 6.5) \\
11-24( \pm 6.5)\end{array}$ \\
\hline IBM PS/2 Model 30286 & $\begin{array}{l}\text { Viglen keyboard DFK2020UK } \\
\text { IBM PS/2 keyboard } 1391406 \\
\text { MEL response box Version } 5.0\end{array}$ & $\begin{array}{l}\mathrm{u} \\
\mathrm{k} \\
\mathrm{l}\end{array}$ & $\begin{array}{l}5,000 \\
5,000 \\
5,000\end{array}$ & $\begin{array}{l}0 \\
3 \ddagger \\
4 \$\end{array}$ & $\begin{array}{l}14.1 \\
16.8 \\
20.0\end{array}$ & $\begin{array}{l}0.6 \\
3.1 \\
1.5\end{array}$ & $\begin{array}{l}12-15( \pm 1.5) \\
11-22( \pm 5.5) \\
18-23( \pm 2.5)\end{array}$ \\
\hline
\end{tabular}

Note - Stimuli were displayed in middle of screen, i.e., START LINE 13 in MEL. Outliers and error trials were omitted from calculation of summary statistics. *Two trials coded as nonresponses ( RESPAC $=2$ ), one outlier of $433 \mathrm{msec}$. †ne trial coded as nonresponse. †Three trials coded as nonresponses. Four trials coded as out-of-range responses.

The lower half of Table 1 shows data from three quite different types of response device-namely, the MEL response box (Version 5.0), an IBM PS/2 1391406 keyboard, ' and the Viglen DFK2020UK keyboard. Each was tested with an IBM PS/2 Model 30286 computer. The results indicated that both the MEL response box and the Viglen DFK2020UK keyboard had a lesser degree of random error than the IBM 1391406 keyboard (IBM keyboard: $99.8 \%$ data within $\pm 5 \mathrm{msec}$ of $16 \mathrm{msec}$ ). It is also of interest to note that the distribution of response times varied across the different response devices (see Figure 2). For example, the distribution of data from the Viglen DFK2020UK keyboard was unimodal, with relatively low variance and high kurtosis, which would seem to be desirable characteristics for a response device used for RT measurement.

Our estimated random error of $\pm 5 \mathrm{msec}$ for $99.8 \%$ data from the IBM 1391406 keyboard is the same as that found by Segalowitz and Graves (1990) for the PS/2. Segalowitz and Graves assessed keyboard response times using an "auditory reaction time test program," and estimated that the mean delay ${ }^{2}$ of the IBM PC keyboard used with an IBM PS/2 computer was about $10 \mathrm{msec}$ with a random error of $\pm 5 \mathrm{msec}$. Consequently, we were encouraged that our data appear to confirm that random timing errors in keyboards are relatively low. Thus, the keyboard seems acceptable as a response device for MEL if there are sufficient trials to take account of the error variance (Segalowitz \& Graves, 1990). However, in future research where there is a relatively small number of trials in each condition and where the effect size is expected to be small, it would seem preferable to use the MEL response box (or a keyboard with a known small random error, such as the Viglen DFK2020UK) rather than an unevaluated keyboard.

In conclusion, we have found MEL to be an invaluable tool in our research, and it has complemented our work with more traditional tachistoscopic methods of presenting brief visual displays. However, it is advisable that researchers should check the timing of brief screen displays. Our research has indicated that it is advisable to use WAITTOP, but not ERASE, instructions on the stimulus and mask FRAMES (as recommended in the MEL manual), and also to use DURATION instructions, which are at least $1 \mathrm{msec}$ less than the multiple of the refresh interval of the monitor. Also, investigation of response timing issues indicates that the MEL response box may be preferable to keyboards for certain tachistoscopic tasks where error variance needs to be kept to a minimum.

\section{REFERENCES}

Bradley, B. P., Mogg, K., Millar, N., Bonham-Carter, C., Fergusson, E., Jenkins, J., \& PARR, M. (1995). Attentional biases for emotional facial expressions. Manuscript submitted for publication.

Bradley, B. P., MogG, K., Millar, N., \& White, J. (1995). Selective processing of negative information: Effects of clinical anxiety, concurrent depression, and awareness. Journal of Abnormal Psychology, 104, 532-536.

Bradley, B. P., MogG, K., \& Williams, R. (1994). Implicit and explicit memory for emotional information in non-clinical subjects. Behaviour Research \& Therapy, 32, 65-78.

Felsten, G., \& Wasserman, G. S. (1980). Visual masking: Mechanisms and theories. Psychological Bulletin, 88, 329-353.

Mogg, K., Bradley, B. P., \& Williams, R. (1995). Attentional bias in anxiety and depression: The role of awareness. British Journal of Clinical Psychology, 34, 17-36.

Mogg, K., Bradley, B. P., Williams, R, \& Mathews, A. (1993). Subliminal processing of emotional information in anxiety and depression. Journal of Abnormal Psychology, 102, 304-311.

SCHNEIDER, W. (1988). Micro Experimental Laboratory: An integrated system for IBM PC compatibles. Behavior Research Methods, Instruments, \& Computers, 20, 206-217.

SCHNEIDER, W. (1990). MEL users' guide: Computer techniques for real time experimentation. Pittsburgh: Psychology Software Tools.

Segalowitz, S. J., \& Graves, R. E. (1990). Suitability of the IBM XT, $\mathrm{AT}$, and PS/2 keyboard, mouse, and game port as response devices in reaction time paradigms. Behavior Research Methods, Instruments, \& Computers, 22, 283-289.

TRAVIS, D. (1991). Effective colour displays: Theory and practice. London: Academic Press.

\section{NOTES}

1. On the IBM PS/2 1391406 keyboard, the keypress simulator was connected to the input of the keyboard matrix decoder, which is effectively the same as connecting the simulator to the key switch contacts (it is difficult to get access to the latter on this keyboard without causing damage).

2. Our estimated mean interval of $17 \mathrm{msec}$ between the start of the stimulus FRAME and the detection of a keyboard response for the IBM PS/2 keyboard and computer closely agrees with Segalowitz and Graves's (1990) estimate of $10 \mathrm{msec}$ for mean keyboard delay, given that we were assessing response times to a stimulus in the middle of the screen, which would explain an additional mean delay of about $7 \mathrm{msec}$ due to the raster scan transit time.

(Manuscript received July 5, 1994; revision accepted for publication October 29,1994 .) 